\title{
The effect of boundaries on the ion acoustic beam-plasma instability in experiment and simulation.
}

\author{
Christopher Rapson, ${ }^{1, *}$ Olaf Grulke, ${ }^{2}$ Konstantin Matyash,${ }^{3}$ and Thomas Klinger ${ }^{2,3}$ \\ ${ }^{1}$ Max Planck Institute for Plasma Physics, \\ Boltzmannstr. 2, D-85748 Garching, Germany \\ ${ }^{2}$ Max Planck Institute for Plasma Physics, \\ Wendelsteinstr. 1, 17491 Greifswald, Germany \\ ${ }^{3}$ Institut für Physik, Ernst-Moritz-Arndt Universität, \\ Domstr. 10a, 17489 Greifswald, Germany
}

\begin{abstract}
The ion acoustic beam-plasma instability is known to excite strong solitary waves near the Earth's bowshock. Using a Double Plasma experiment, tightly coupled with a 1-dimensional Particle-in-Cell simulation, the results presented here show that this instability is critically sensitive to the experimental conditions. Boundary effects, which do not have any counterpart in space or in most simulations, unavoidably excite parasitic instabilities. Potential fluctuations from these instabilities lead to an increase of the beam temperature which reduces the growth rate such that non-linear effects leading to solitary waves are less likely to be observed. Furthermore, the increased temperature modifies the range of beam velocities for which an ion acoustic beam plasma instability is observed.
\end{abstract}

Keywords: beam plasma instability, ion acoustic wave, VINETA, particle-in-cell simulation, double plasma, sheath

${ }^{*}$ Electronic address: chris.rapson@ipp.mpg.de 


\section{INTRODUCTION}

Space plasmas are generally considered collisionless, which immediately implies two properties: the dynamics is dominated by collective kinetic physics [1] and the velocity distribution is often non-Maxwellian. A beam propagating as a minority population is a particularly strong deviation from a Maxwellian distribution, and has been observed to excite kinetic instabilities in different regions of space. For example at the boundaries of supernova remnants $[2,3]$, reconnection events [4], bowshocks [5, 6] or coronal mass ejections [7-9]. Due to their fast growth rate and the absence of collisional damping, beam-plasma instabilities in space are generally observed in a state of non-linear saturation, either as turbulence [10, 11] or as solitary waves $[12,13]$. In the case of an ion beam moving with the ion acoustic velocity, as observed in the earth's bowshock [5], the solitary waves are associated with anomalous resistivity [14-16] which makes this instability particularly interesting.

The linear theory for the Ion Acoustic Beam-Plasma Instability (IABPI) is well understood for simplified cases, and will be outlined in section II. To investigate non-linear effects and to overcome diagnostic difficulties, simulations have been a useful tool [e.g. 17-21] to the point where comparisons with naturally observed structures can be made with remarkable accuracy [22, 23].

Complementary investigations of the IABPI in laboratory experiments have proven more challenging. Diagnostics for ion phase space are notoriously difficult $[24,25]$ and the instability itself is sensitive to experimental conditions. While in space the instability is almost exclusively observed in a fully non-linear saturated state, there appears to be only one published observation from a laboratory experiment of solitary waves driven by an ion beam in a stationary state [26]. Also, one of the more common experiments relates to the dependence of the instability on the beam velocity. As shown in table I, large variation has been found. This paper presents evidence from experiments in the linear plasma device VINETA [27], and numerical studies using a particle-in-cell (PIC) simulation which explicitly includes boundaries [28] to show that the boundaries themselves can be responsible for both of these observations.

In the work presented here, and all of the experiments in table I, a Double Plasma (DP) setup is used to generated the ion beam. As the name suggests, a DP consists of two thermionic plasmas, separated by a strongly negative dividing grid. Electrons can not 
TABLE I: Lower and upper limits for the ion beam velocity in terms of the ion acoustic velocity, for which ion beam driven acoustic instabilities have been observed in experiments.

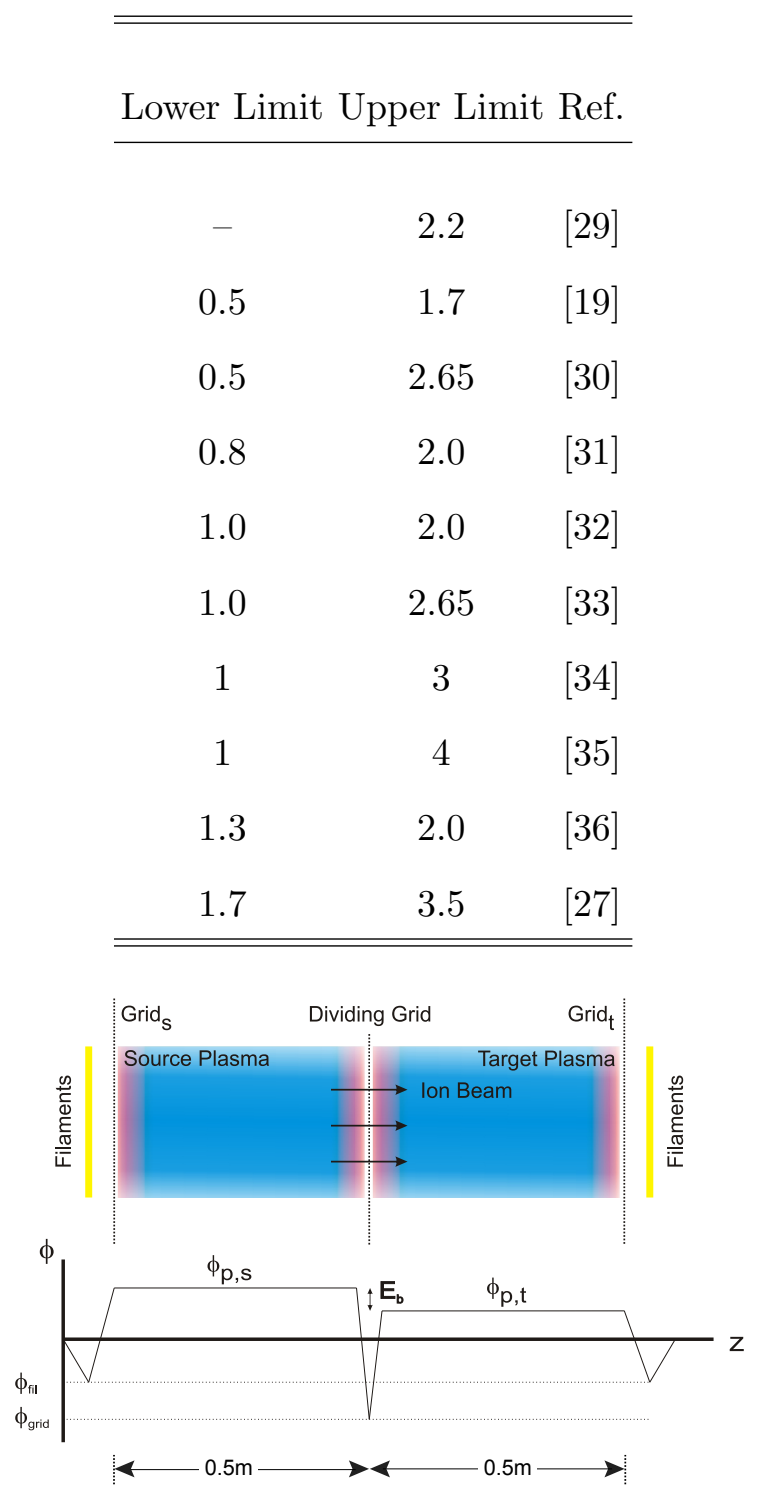

FIG. 1: Schematic showing the Double Plasma setup, including the potential profile.

pass through the grid, which allows the potential of the plasmas $\left(\phi_{p, s}\right.$ and $\left.\phi_{p, t}\right)$ to be set independently. In each case, the plasma potential is dictated by the potential of the most positive boundary (plus a small offset for the sheath potential). The setup is represented in the diagram in Fig. 1. Ions passing from the source to the target plasma are accelerated as they approach the central grid, and decelerated again after passing through it. Their energy $E_{b}$ when they exit the sheath is given by the difference in the two plasma potentials. 

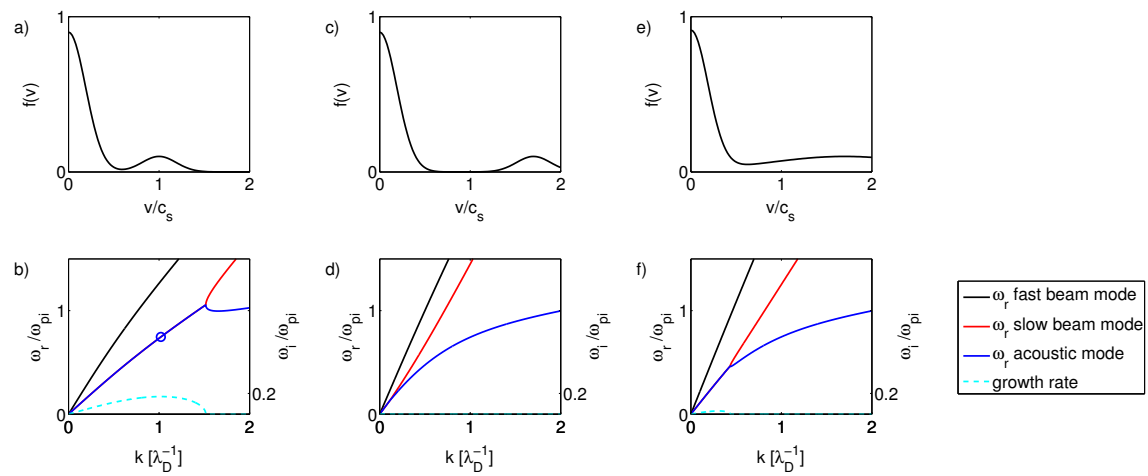

FIG. 2: Distribution functions (top row) and dispersion relations (bottom row) for different beam properties: a\&b: $v_{b}=c_{s}, T_{b}=T_{i}=0.1 \mathrm{eV} . \mathrm{c} \& \mathrm{~d}: v_{b}=1.7 c_{s}, T_{b}=T_{i}=0.1 \mathrm{eV}$. e\&f: $v_{b}=1.7 c_{s}$, $T_{b}=20 T_{i}=2.0 \mathrm{eV}$.

\section{DISPERSION RELATION}

Beam-plasma instabilities are collective, kinetic instabilities. Waves with a phase velocity $v_{p h}=\frac{\omega}{\vec{k}}$ will be resonantly excited when $\left.\frac{\partial f}{\partial \vec{v}}\right|_{\vec{v}_{p h}}>0$. $\omega$ is the wave frequency, $\vec{k}$ the wavenumber and $f(\vec{v})$ is the particle velocity distribution function. If the distribution function is reduced to a double Maxwellian distribution for the ions, and a single Maxwellian for the electrons, and only one dimension is considered such that vectors collapse to scalars, the dispersion relation for the IABPI can be written as $[32,37]$

$$
1+\frac{1}{k \lambda_{D}}-\frac{1}{2} Z^{\prime}\left[\frac{\omega}{k c_{s}}\left(\frac{m_{e}}{m_{i}}\right)^{\frac{1}{2}}\right]-\frac{1}{2} \frac{n_{b} T_{e}}{n_{i} T_{b}} Z^{\prime}\left[\frac{\omega-v_{b} k}{k c_{s}}\left(\frac{T_{e}}{2 T_{i}}\right)^{\frac{1}{2}}\right]=0
$$

where $Z$ is the plasma dispersion function, $m$ is the mass of a species, $v$ its mean velocity, $T$ its temperature and $n$ its density. The relevant species are designated by subscripts $e$ for electrons, $i$ for bulk ions and $b$ for beam ions. $\lambda_{D}=\sqrt{\frac{\epsilon_{0} T_{e}}{n_{e} e}}$ is the Debye length and $c_{s}=\sqrt{\frac{e\left(T_{e}+\gamma T_{i}\right)}{m_{i}}} \sim \sqrt{\frac{e T_{e}}{m_{i}}}$ is the ion acoustic velocity. When not used as a subscript, $e$ represents the elementary charge and $\epsilon_{0}$ is the permittivity of free space.

When solving (1) for $\omega$, a complex solution indicates unstable growth. Plotting the solutions for $\omega$ as a function of $k$ allows the dispersion relation to be visualised, as shown in fig. $2 \mathrm{~b}$ for a standard case with $v_{b}=c_{s}, T_{b}=T_{i}=0.1 T_{e}$. The plasma can support three modes in the range of wavenumbers and frequencies shown - the fast beam mode, the slow beam mode and the ion acoustic mode. Each is plotted as a separate line. Where two lines meet $(0<k<1.8)$, the solution is complex-conjugate. Over the same range, the 
TABLE II: Numerical parameters as used for the PIC simulations. Note that the parameters have not been optimised for execution time, since this was not a restricting factor.

\begin{tabular}{ccc}
\hline \hline Parameter Description & Parameter & Normalised setting \\
\hline$d t_{e}$ & electron timestep & $\frac{0.1}{\omega_{p e}}$ \\
$d t_{i}$ & ion time step & $\frac{0.4}{\omega_{p e}}$ \\
$d t_{e, \text { coll }}$ & electron inelastic collision time step & $\frac{5}{\omega_{p e}}$ \\
$d t_{i, \text { coll }}$ & ion inelastic collision time step & $\frac{8}{\omega_{p e}}$ \\
$d x$ & spatial resolution & $0.3 \lambda_{D}$ \\
$\frac{m_{i}}{m_{e}}$ & mass ratio & $\frac{m_{A r}}{m_{e}}=73500$ \\
$\frac{n_{s p}}{\lambda_{D}}$ & superparticle density & 30 \\
\hline \hline
\end{tabular}

imaginary component of the solution can be seen in cyan. The corresponding distribution function is shown in fig. 2a. The bulk has zero mean velocity and the beam is visible as a "bump on tail" centred at $1 c_{s}$. Note that the gradient $\frac{\partial f}{\partial v}$ is steepest for $v=0.81 c_{s}$, which corresponds to the phase velocity with the maximum growth rate, marked by the circle in fig. $2 \mathrm{~b} v_{p h}=\frac{0.746 \omega_{p i}}{1.02 \lambda_{D}^{-1}}=0.73 c_{s}$, where the ion plasma frequency $\omega_{p i}=\sqrt{\frac{n e^{2}}{m \epsilon_{0}}}$.

As the velocity is decreased, the beam is absorbed into the bulk distribution, $\frac{\partial f}{\partial v}<0$ over all velocities. Trivially, the instability no longer appears. At higher beam velocities, $\frac{\partial f}{\partial v}<0$ only for $v>c_{s}$, which is the maximum phase velocity for an ion acoustic wave. This case is shown in fig. 2c, with the corresponding dispersion relation in fig. 2 d. The modes are now clearly separated, and have no complex component.

Fig 2e and $\mathrm{f}$ show a similar situation, but now with a higher beam temperature. For moderate $v_{b} \sim c_{s}$, the gradient $\frac{\partial f}{\partial v}$ would be reduced, and hence the growth rate would also be smaller. However, for higher $v_{b}$ the growth rate actually increases with increasing beam temperature. As shown in the figure, the region where $\frac{\partial f}{\partial v}>0$ now extends below $c_{s}$ and now the dispersion relation predicts a finite growth rate, albeit smaller than in the first example. 

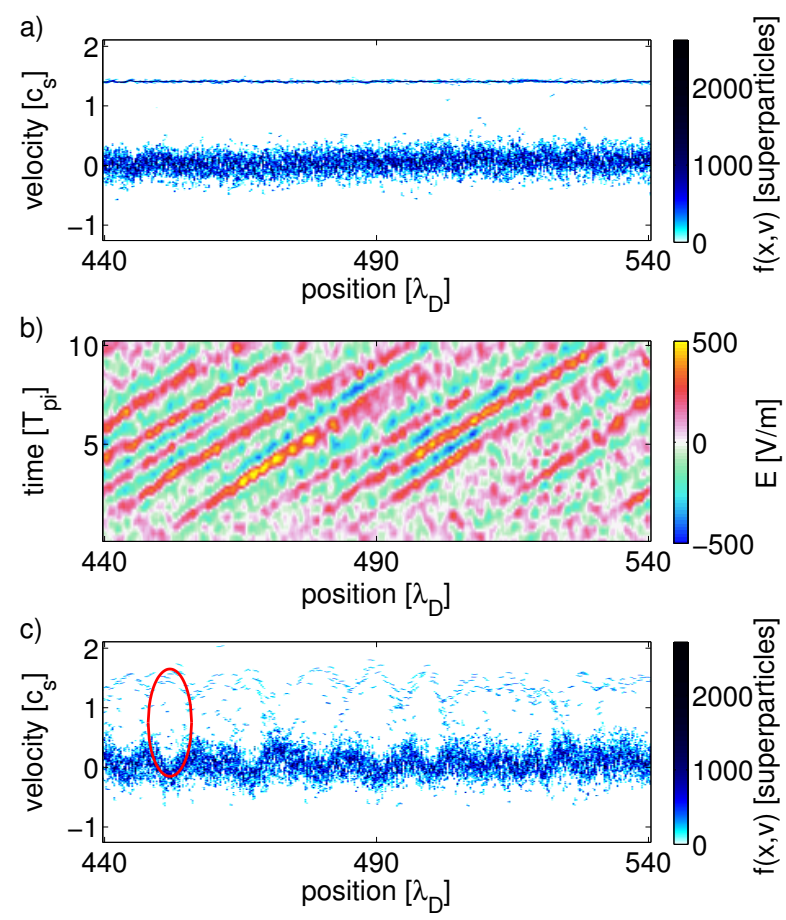

FIG. 3: Results from a simulation with initial beam and bulk distribution independent of position. a) Ion phase space $f(x, v)$ at time $=0$. b) Electric field as a function of position and time, with time in units of the ion plasma period $T_{p i}=\frac{2 \pi}{\omega_{p i}}$. c) Ion phase space $f(x, v)$ at a time where non-linear effects are particularly prevalent.

\section{SIMULATION}

Particle-in-Cell (PIC) simulations allow a full representation of ion phase space, which is necessary to properly investigate kinetic instabilities. For the results presented here, a 1-dimensional (1-D) PIC code [28] was used, with the special feature that boundaries and the associated sheaths are self-consistently modelled. Although neither the earth's bowshock nor the laboratory experiments are 1-D systems, it is assumed that a 1-D model is appropriate for planar waves where the wavelength in the direction parallel to the beam propagation is much shorter than both the perpendicular wavelength $\left(k_{\|} \gg k_{\perp}\right)$ and the perpendicular gradient length of plasma parameters. For linear laboratory experiments, this is approximately the width of the plasma column $\left(k_{\|} \gg \frac{2 \pi}{\text { plasma width }}\right)$. The simple case described in the previous section can be reproduced with no boundaries and a homogeneous velocity distribution. (Homogeneous in the sense that it is independent of position.) Fig. 3a shows the initial conditions in phase space co-ordinates where most ions are in the bulk 


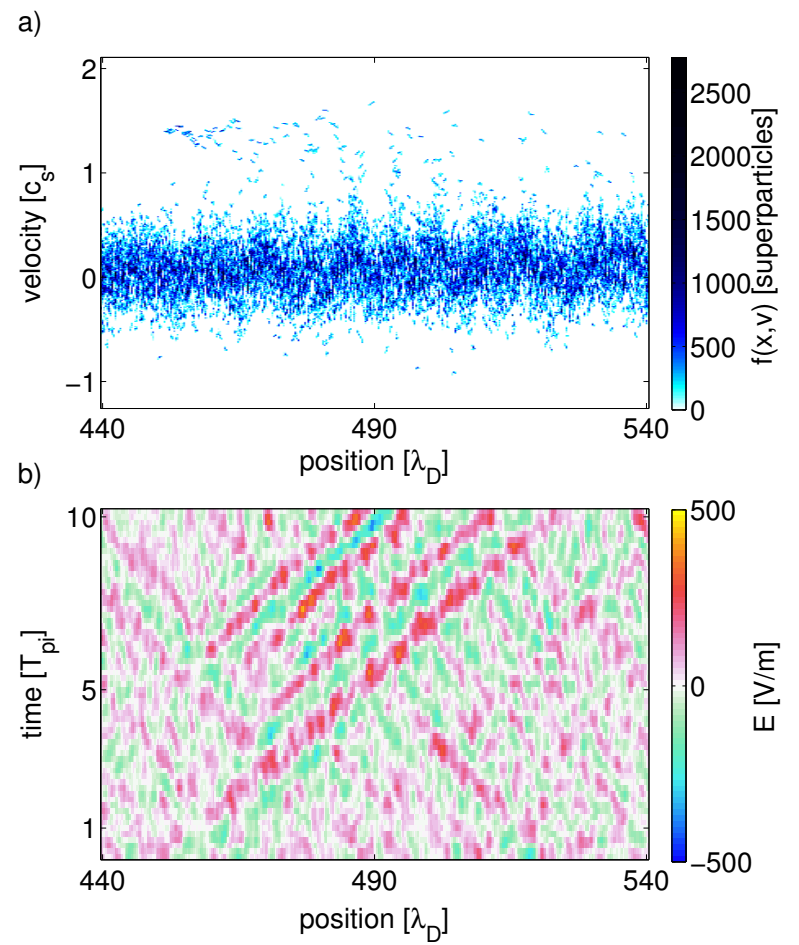

FIG. 4: Results from a simulation with a beam injected into a homogeneous bulk plasma. a) Ion phase space $f(x, v)$. b) Electric field as a function of position and time.

population, centered on zero velocity, and a beam population with $10 \%$ of the bulk density is moving with a velocity of $v_{b}=1.4 c_{s}$. Relevant numerical constants are listed in table II. This kind of simulation has been conducted many times before, and as expected, the ion acoustic wave is excited and grows to a significant amplitude. Fluctuations in the plasma potential create electric fields of up to $500 \mathrm{Vm}^{-1}$ as shown in fig. $3 \mathrm{~b}$, which translates to strong potential fluctuations on the order of the electron temperature. The mode growth saturates non-linearly in so-called "phase space holes". One of a chain of phase space holes has been highlighted in fig. 3c. Phase space holes are aligned with regions of negative potential and trap ions into closed trajectories (ellipses in 1-D phase space). Since these holes have a propensity to merge, a chain can develop into solitary waves as observed in space, providing collisional damping is small.

To tailor the simulation closer to the experiment, a source is added injecting ions at $x=453 \lambda_{D}$ and moving to the right. To prevent a discontinuity in the density, bulk ions are removed from this position. The injected beam velocity and temperature are the same as before. Fig. 4 depicts the results of this simulation, after the beam has been injected 
TABLE III: Collision frequencies for the simulated plasma conditions: $n_{e}=n_{i}=10^{15} \mathrm{~m}^{-3}, T_{e}=$ $2 \mathrm{eV}, T_{i}=0.1 \mathrm{eV}$, neutral pressure $=0.1 \mathrm{~Pa}$.

\begin{tabular}{cc}
\hline \hline Species & Collision Frequency (normalised to the ion plasma frequency) \\
\hline ion-ion & $3.2 \times 10^{-3}$ \\
ion-neutral & $6.8 \times 10^{-3}$ \\
charge exchange & $9.5 \times 10^{-3}$ \\
ion-electron & $1.4 \times 10^{-2}$ \\
\hline \hline
\end{tabular}

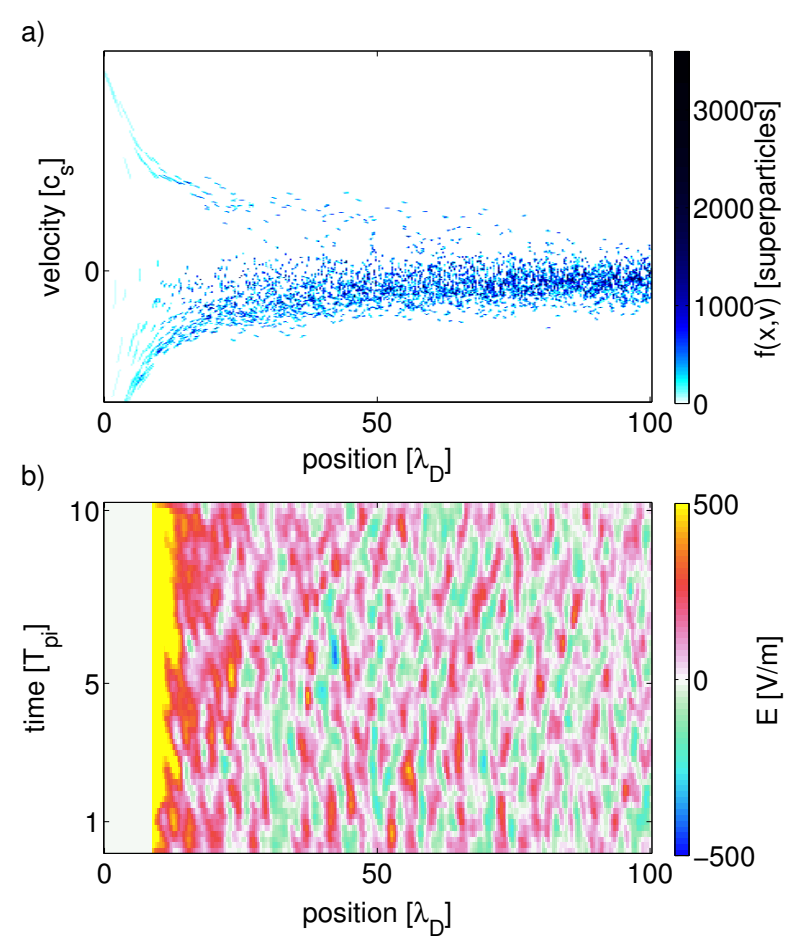

FIG. 5: Results from a simulation with a beam injected into the boundary sheath of a bulk plasma. a) Ion phase space $f(x, v)$. b) Electric field as a function of position and time.

for some time and the instability has reached a sort of steady state. The instability can be observed to develop in space from the point of injection at the left to non-linear saturation at the right in just a few wavelengths. Fig. 4b shows that the amplitude of electric field fluctuations is approximately $400 \mathrm{Vm}^{-1}$ for $x \sim 480 \lambda_{D}$ before being damped by collisions. The collision frequencies for these conditions are listed in table III. 
a)
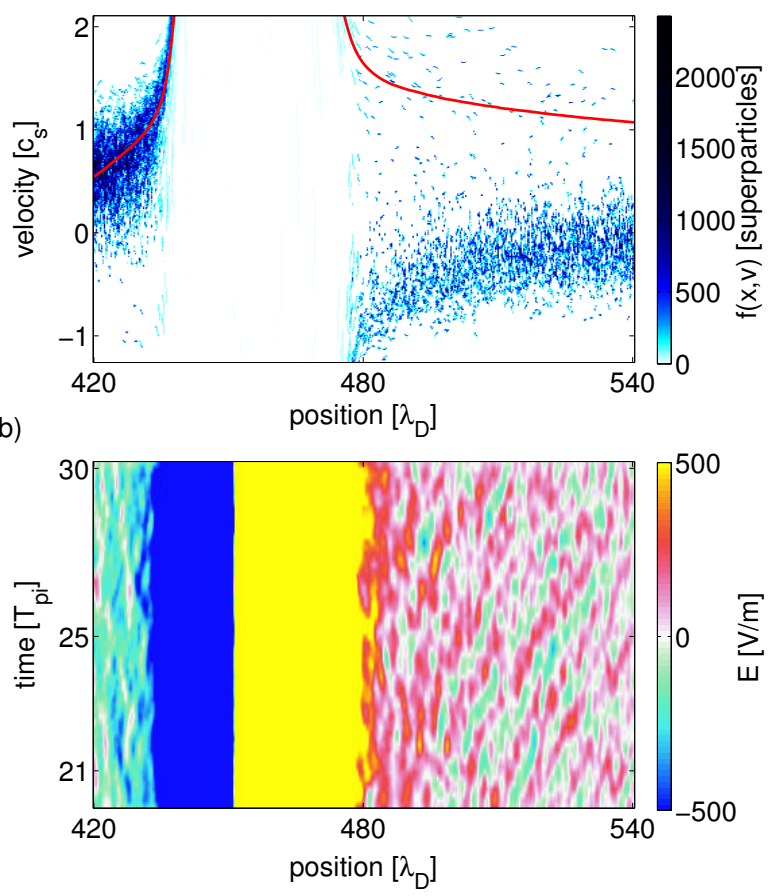

FIG. 6: Results from a simulation with a beam driven by the potential difference between source and target plasmas. a) Ion phase space $f(x, v)$. b) Electric field as a function of position and time.

If the point of injection is moved to $x=0$, we can start to investigate the role of the boundary. Particles reaching the boundary are removed from the simulation, which leads self-consistently to the formation of a sheath. The injected beam velocity must be adjusted for the potential gradient in the sheath. The velocity of injected particles is set so that the beam velocity is the same as before at $x=30 \lambda_{D}$, i.e. when the bulk velocity approaches zero. Although the beam is injected with the same velocity spread as before, fig. 5a shows the beam spreading in velocity space as it decelerates. To some extent, this is simply due to the quadratic relationship between velocity and energy; all particles lose the same energy in the sheath, but for the slower ones this equates to a larger change in velocity. This effect is added to by small angle deflections from small local and transient changes in the potential gradient. While the beam is decelerating in the sheath, the velocity difference between bulk and beam is too large for an IABPI. Starting from $x \sim 30 \lambda_{D}$, growth of an ion-acoustic wave is visible, but the growth rate is reduced due to the increased beam temperature, and phase space holes are not observed. Fig. 5b shows that electric field fluctuation amplitudes are less coherent and reduced to less than $200 \mathrm{Vm}^{-1}$. 
Finally, the simulation is reconfigured to emulate a double plasma. The potential at a central point $x=453 \lambda_{D}$ is fixed strongly negative corresponding to a biased grid in the plasma. To avoid numerical instability from trapped ions, a sink is created by removing some ions from the region close to the grid every time-step. The simulation boundaries are set to give the desired beam velocity $\phi_{\text {source }}=\phi_{\text {target }}+\frac{\frac{1}{2} m v_{b}^{2}}{q}$ for a beam propagating through the central grid from the source into the target plasma. To provide a similar beam density to the previous cases, the source density is increased with respect to the target.

The result of this simulation is shown in fig. 6. In fig 6a the acceleration and deceleration of the ions near the grid can be seen. The red line indicates the expected ion trajectory based on the time averaged potential. Initially, ions approaching the grid from the source plasma follow this trajectory fairly closely, and simultaneously reduce their velocity spread (i.e. the reverse of what was explained for decelerating ions in fig. 5a). However, when exiting the sheath on the other side, the beam has been spread dramatically in velocity space. Clearly, the beam ions do not follow the trajectory given by the time-averaged potential, but are dispersed by fluctuations of the potential.

Potential fluctuations in sheaths are common. Particularly for the sheaths surrounding a grid in a double plasma, the sheath instability is well known in experiments [38, 39] and has been reproduced in simulation [40]. The results from Rohde's Vlasov simulation showing bunching of ions within the sheath and in extreme cases expulsion of 'tongues' of ions into the target plasma could be reproduced in the course of the current work (not shown).

Those potential fluctuations lead to an increase of the beam temperature, and a reduction of the growth rate for the IABPI. Fig $6 \mathrm{~b}$ shows that the amplitude of the electric field fluctuations is less than $200 \mathrm{Vm}^{-1}$ despite the basic parameters (beam density and velocity) being the same as the other simulation runs.

From the four cases simulated here, the biggest difference is observed when the injection location is changed from a homogeneous background plasma to the boundary. Holding all other variables constant, this shows that the increase in ion beam temperature associated with sheath dynamics are decisive for the evolution of the IABPI.

Having developed a simulation which closely replicates a DP, the IABPI can be studied in detail by varying some of the variables in the simulation. Shown in the following are examples of density fluctuations for varying beam velocity. By taking the two-dimensional Fast Fourier Transform (2D-FFT), the fluctuations can be converted to frequency space 

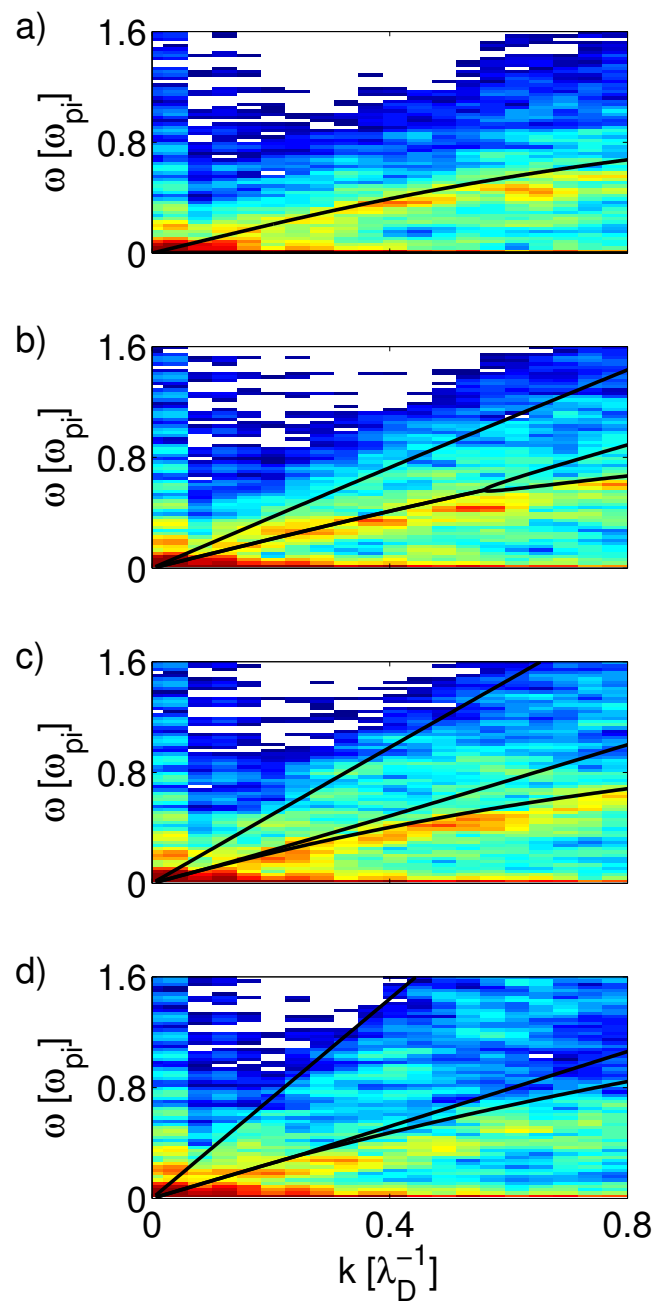

FIG. 7: Plots of frequency against wavenumber from two dimensional Fourier spectra of the potential fluctuations from a simulation of a double plasma experiment. Examples are shown for the following beam velocities: a) $0.2 c_{s}$, b) $1.2 c_{s}$, c) $1.8 c_{s}$, d) $2.5 c_{s}$.

$(\omega, k)$ and compared to the analytical dispersion relation. Since the simulation provides full access to the ion phase space, in particular the beam temperature, all of the input parameters are available. Fig. 7 shows a series of comparisons for varying beam velocities. In each case, the analytical dispersion relation, shown in black, corresponds very well with the amplitude patterns from the simulation. At low velocities, as in fig. 7a, there is no instability, and only the background noise is observed. For moderate velocities, fig $7 \mathrm{~b}$ and c, the instability is visible as an increased amplitude in the same region as predicted by the dispersion relation. At high velocities two independently propagating modes can be observed fig. $7 \mathrm{~d}$, corresponding to the beam mode (between the upper two black lines) and 


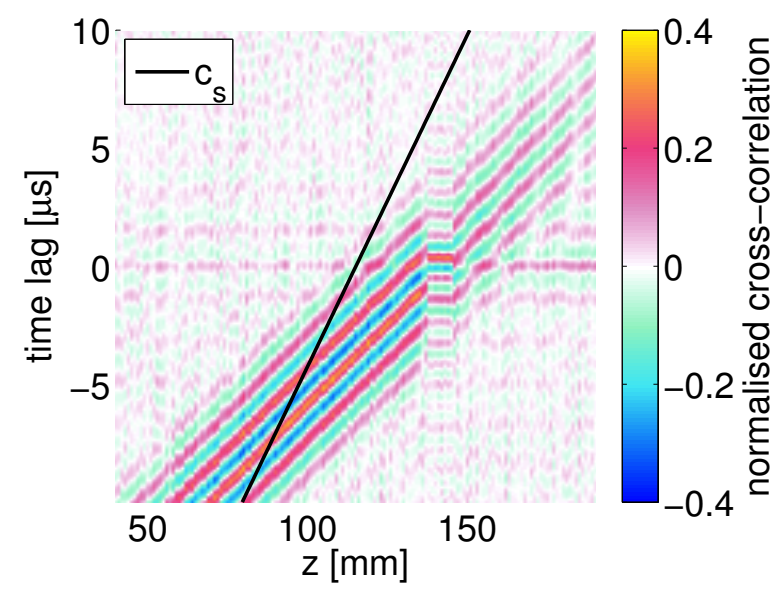

FIG. 8: Cross correlation between two probes, one stationary and one which was moved along the beam propagation direction. The $\mathrm{z}$ axis gives the position of the moveable probe relative to the central grid.

the acoustic mode. The beam modes are however not unstable and hence have a lower amplitude. A discussion of the velocities at which the instability appears will be made in conjunction with experimental results at the end of section IV.

\section{EXPERIMENTAL RESULTS}

Experiments were conducted in the linear plasma device VINETA [41], which was converted to a double plasma setup. The densities and potentials of each plasma could be set (almost) independently, such that an ion beam with the desired density and velocity propagated from the source to the target plasma. An electron density of $n_{e}=2.9 \times 10^{14} \mathrm{~m}^{-3}$ and electron temperature of $T_{e}=4.0 \mathrm{eV}$ were measured in the target plasma with a Langmuir probe. The plasma was not magnetised for these experiments. The beam velocity and density were measured with a Retarding Field Energy Analyser (RFEA) [42]. Measurements of the floating potential fluctuations were taken with a pair of electrostatic probes - one stationary and one which was moved along the direction of beam propagation. The signals were high-pass filtered and amplified using amplifiers with a high bandwidth to extract fluctuations on the order of the ion plasma frequency.

Fig 8 shows the space-time evolution of the cross correlation amplitude between the floating potential fluctuations. The $z$ co-ordinate is the distance from the dividing grid. 

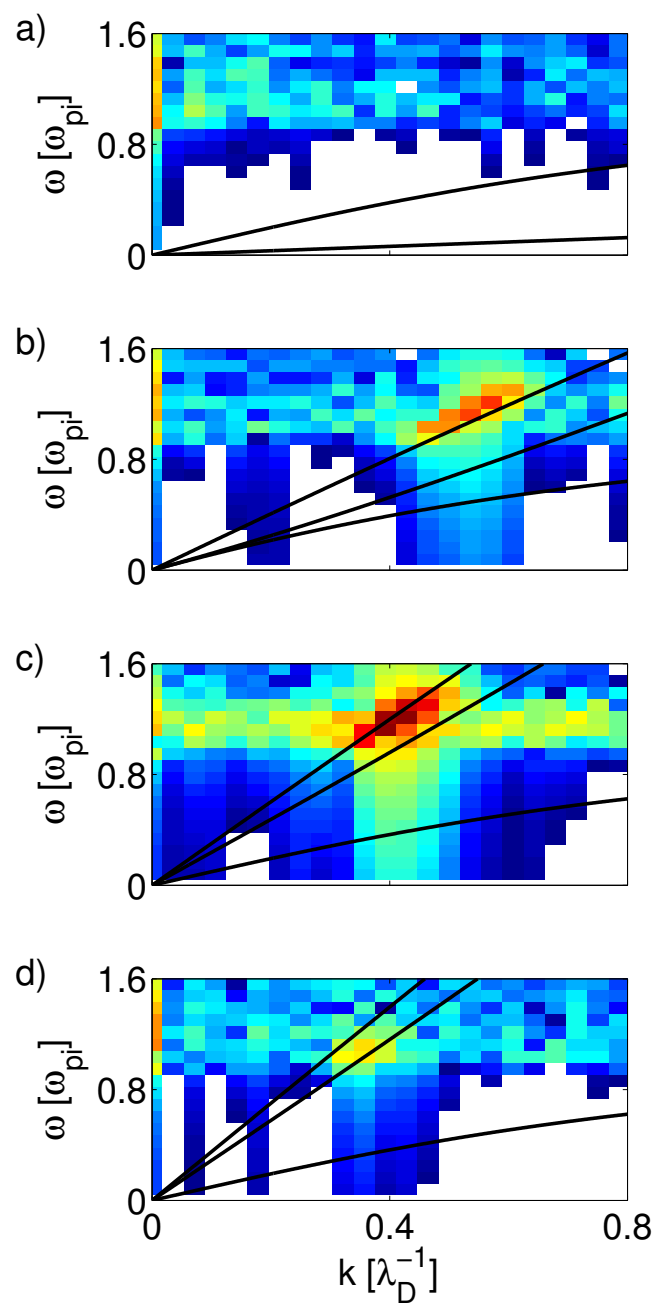

FIG. 9: Plots of frequency against wavenumber from two dimensional Fourier spectra of the crosscorrelation of floating potential fluctuation measurements by two probes, which is compared to the analytical dispersion (black lines). Examples are shown for the following beam velocities: a) $0.0 c_{s}$, b) $1.7 c_{s}$, c) $2.7 c_{s}$, d) $3.2 c_{s}$.

The diagonal pattern shows a propagating wave, whose phase velocity is close to the ion acoustic velocity as indicated by the black line. The wave has been excited by an ion beam with velocity $v_{b}=2.2 c_{s}$. A sheath instability was also observed, with almost the same frequency, but much faster propagation: $k \sim 0$. The discontinuity at $x \sim 140 \mathrm{~mm}$ is caused by the proximity of the two probes to each other.

Similar to the simulation results, the experimental measurements can be compared with the dispersion relation by taking the 2D-FFT of the cross-correlation. Fig 9 shows some examples, starting with a situation where there is no beam and no instability in frame (a). 


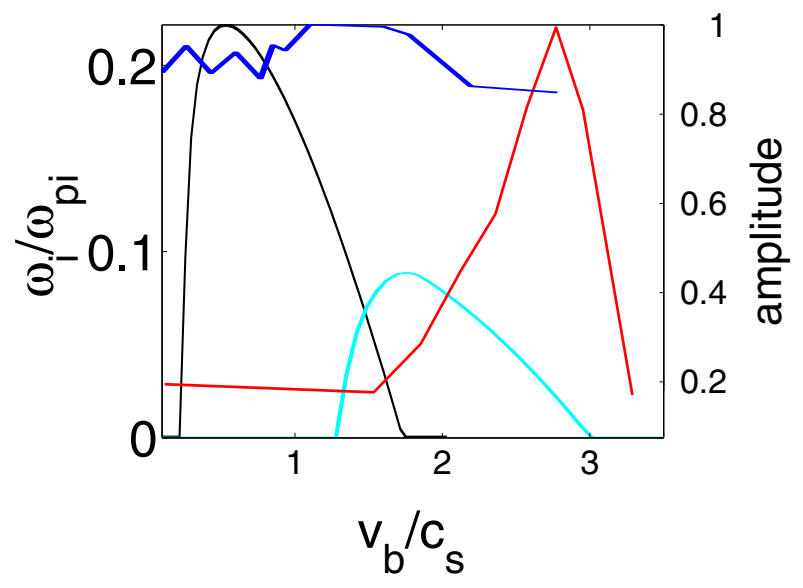

FIG. 10: Plots of growth rate against beam velocity from the analytical dispersion relation, with $T_{b}=T_{i}$ in black and $T_{b}=6 T_{i}$ in cyan. This is compared with observed amplitudes from simulation (blue) and experiment (red) which have been normalised to the maximum observed amplitude.

The axes have been normalised for $\lambda_{D}=0.9 \mathrm{~mm}$ and $f_{p i}=\frac{\omega_{p i}}{2 \pi}=0.6 \mathrm{MHz}$. In (b), there is a low amplitude instability excited by a beam with velocity $v_{b}=1.7 c_{s}$. Frame (c) shows a faster beam with the maximum amplitude observed in this experiment. Still, no non-linear effects were detected. In the final frame, the beam is too fast to generate an instability. In all cases, the black lines show the analytical dispersion relation, whereby it should be noted that despite attempts with RFEA and Laser Induced Fluorescence [42], it was not possible to obtain an accurate measurement of the ion beam temperature. In the absence of a reliable measurement, it was assumed that the beam ions retained the same temperature as in the source, which was measured to be $0.13 \mathrm{eV}$. Given this and other experimental uncertainties, the quantitative agreement between the measured and expected dispersion relation is reasonable. The qualitative agreement is good, for example the trend of shifting the instability to shorter wavenumbers (higher phase velocities) at higher beam velocities which can be seen by comparing the two unstable cases in fig. 9 .

Considering now the strength of the instability with respect to the beam velocity, fig. 10 compares the maximum amplitude from simulation and experiment with growth rates from analytical dispersion relations. The maximum amplitude is used as a proxy for the growth rate since growth is limited by collisions and difficult to evaluate. The figure shows that for both simulation in blue and experiment in red, the range of velocities over which an instability occurs is higher than $0.3 c_{s}<v_{b}<1.7 c_{s}$ as would be expected if one assumed 
$T_{b}=T_{i}$ (the black curve). For the simulation, the noise level is relatively high even with no beam. Nevertheless, an amplitude increase can be observed for $1.0 c_{s}<v_{b}<2.1 c_{s}$. In the experiment, the range is $2.0 c_{s}<v_{b}<2.8 c_{s}$. When $T_{b}$ is increased, the growth rate drops, but the unstable range of velocities is shifted to higher velocities, thereby obtaining a good agreement with the results from simulation or experiment. Since the sheath instability depends on the same parameters as the IABPI - densities and relative potential of the source and target plasmas - as well as neutral pressure and grid voltage, it is difficult to predict exactly what the effect of this boundary on the IABPI will be. The interaction between the two instabilities is far from trivial. The reason for the remaining difference between simulation and experiment is not clear, but could easily be explained by 2-D or 3-D effects, small differences in the sheath instability or numerical effects, among a range of other possibilities.

\section{SUMMARY \& CONCLUSIONS}

The results presented here based on a 1-D PIC simulation and a double-plasma experiment provide evidence that the well known sheath instability causes an increase in beam temperature in double-plasma configurations. The dispersion relation for the IABPI indicates that the increased beam temperature will lead to two effects: a reduction in the growth rate, and a shift to higher values in the range of velocities over which an instability is observed. These two effects could be confirmed by both simulation and experiment.

The PIC simulation resolves the complete ion phase space, such that the increase in ion beam temperature can be clearly visualised and an excellent match with the dispersion relation is obtained. In experiment, it was impossible to resolve the beam temperature, but still the dynamics closely follow the trends of the analytical dispersion relation. The simulation further allows the causality to be resolved in a stepwise progression from the usual spatially homogeneous, temporally evolving IABPI to a model which closely resembles the experimental DP. The instability is mostly unchanged between cases with a spatially homogeneous and a locally injected beam. However, when the injection point is moved to the boundary, the beam spreads in velocity space as it decelerates and traverses the sheath. The amplitude of the electric field fluctuations in this case is reduced from $400 \mathrm{Vm}^{-1}$ to less than $200 \mathrm{Vm}^{-1}$. In the final case, instead of the expected reduction in velocity spread when 
the beam is accelerated by the DP, the beam is scattered by small potential perturbations caused by the sheath instability and $T_{b}$ increases dramatically. The IABPI excited by this warm beam again has an electric field fluctuation amplitude slightly less than $200 \mathrm{Vm}^{-1}$.

The reduced growth rate implies that under these conditions, the IABPI is unlikely to reach amplitudes where non-linear effects become dominant. This may be part of the reason why experimental observations of the non-linear phase of the IABPI have been so scarce. Since ion phase space holes, solitary ion acoustic waves and turbulence are commonly observed near bowshocks and constitute an important component of their physics, these results raise the question whether this particular experimental setup is an appropriate tool for ongoing investigations of the IABPI, despite its popularity and past successes with respect to linear dynamics.

The range of beam velocities over which an IABPI is observed was $1.7 c_{s}<v_{b}<3.0 c_{s}$ in experiment, and $1.0 c_{s}<v_{b}<2.0 c_{s}$ in simulation, both of which are higher than would be expected from the dispersion relation assuming $T_{b}=T_{i}: 0.3 c_{s}<v_{b}<1.5 c_{s}$. The sensitive dependence of the IABPI on $T_{b}$, and in turn of $T_{b}$ on the experimental conditions is offered as one possible explanation for the quantitative difference between simulation and experimental results in this paper. The same reasoning could provide an explanation for the incongruous results presented in the literature and summarised in table I.

If this is the case, then our understanding of the IABPI near the Earth's bowshock is not contradicted by these seemingly disparate results. Given that measurements generally show a cold ion beam near the bowshock, it is reasonable that common estimates for the range of unstable velocities are at the lower end of what has been observed in experiments and close to theoretical estimates for $T_{b}=T_{i}$. The more general message is that all relevant parameters should be considered, even when they are difficult to measure and difficult to control, such as $T_{b}$. In the case of the IABPI, where the boundary plays a role in determining such parameters, this physics must be taken into account when evaluating experimental results and extrapolating to space. 


\section{Acknowledgments}

This project has received funding from the Euratom research and training programme 2014-2018.

[1] F. Scarf and D. Gurnett, "Plasma-wave investigation for Voyager mission," Space Sci. Rev., vol. 21, no. 3, pp. 289-308, 1977.

[2] K. Koyama, R. Petre, E. Gotthelf, U. Hwang, M. Matsuura, M. Ozaki, and S. Holt, "Evidence for shock acceleration of high-energy electrons in the supernova remnant SN1006," Nature, vol. 378, pp. 255-258, NOV 161995.

[3] D. J. Patnaude and R. A. Fesen, "Proper motions and brightness variations of nonthermal X-ray filaments in the Cassiopeia A supernova remnant," Astrophys. J., vol. 697, pp. 535-543, MAY 202009.

[4] J. Drake, M. Swisdak, C. Cattell, M. Shay, B. Rogers, and A. Zeiler, "Formation of electron holes and particle energization during magnetic reconnection," Science, vol. 299, pp. 873-877, FEB 72003.

[5] H. Kucharek, E. Mobius, M. Scholer, C. Mouikis, L. Kistler, T. Horbury, A. Balogh, H. Reme, and J. Bosqued, "On the origin of field-aligned beams at the quasi-perpendicular bow shock: multi-spacecraft observations by CLUSTER," Ann. Geophys., vol. 22, no. 7, pp. 2301-2308, 2004 .

[6] S. Moses, F. Coroniti, C. Kennel, F. Scarf, E. Greenstadt, W. Kurth, and R. Lepping, "High time resolution plasma-wave and magnetic-field observations of the Jovian bow shock," Geophys. Res. Lett., vol. 12, no. 4, pp. 183-186, 1985.

[7] R. Lin, D. Potter, D. Gurnett, and F. Scarf, "Energetic electrons and plasma-waves associated with a solar type-III radio-burst," Astrophys. J., vol. 251, no. 1, pp. 364-373, 1981.

[8] R. Ergun, D. Larson, R. Lin, J. McFadden, C. Carlson, K. Anderson, L. Muschietti, M. McCarthy, G. Parks, H. Reme, J. Bosqued, C. D’Uston, T. Sanderson, K. Wenzel, H. Kaiser, R. Lepping, S. Bale, P. Kellogg, and J. Bougeret, "Wind spacecraft observations of solar impulsive electron events associated with solar type iii radio bursts," Astrophys. J., vol. 503, pp. 435-445, AUG 101998. 
[9] M. J. Reiner, K. Goetz, J. Fainberg, M. L. Kaiser, M. Maksimovic, B. Cecconi, S. Hoang, S. D. Bale, and J. L. Bougeret, "Multipoint observations of solar type III radio bursts from STEREO and WIND," Sol. Phys., vol. 259, pp. 255-276, OCT 2009.

[10] H. Matsumoto, H. Kojima, T. Miyatake, Y. Omura, M. Okada, I. Nagano, and M. Tsutsui, "Electrostatic solitary waves (ESW) in the magnetotail - BEN wave-forms observed by GEOTAIL," Geophys. Res. Lett., vol. 21, pp. 2915-2918, DEC 151994.

[11] P. Decreau, P. Fergeau, V. Krasnoselskikh, E. Le Guirriec, M. Leveque, P. Martin, O. Randriamboarison, J. Rauch, F. Sene, H. Seran, J. Trotignon, P. Canu, N. Cornilleau, H. de Feraudy, H. Alleyne, K. Yearby, P. Mogensen, G. Gustafsson, M. Andre, D. Gurnett, F. Darrouzet, J. Lemaire, C. Harvey, P. Travnicek, and W. Expt, "Early results from the Whisper instrument on CLUSTER: an overview," Ann. Geophys., vol. 19, pp. 1241-1258, OCT-DEC 2001.

[12] R. Bostrom, G. Gustafsson, B. Holback, G. Holmgren, H. Koskinen, and P. Kintner, "Characteristics of solitary waves and weak double-layers in the magnetospheric plasma," Phys. Rev. Lett., vol. 61, pp. 82-85, JUL 41988.

[13] S. Bounds, R. Pfaff, S. Knowlton, F. Mozer, M. Temerin, and C. Kletzing, "Solitary potential structures associated with ion and electron beams near 1 R-E altitude," J. Geophys. Res. Lett. - Space Phys., vol. 104, pp. 28709-28717, DEC 11999.

[14] S. Hamberger and M. Friedman, "Electrical conductivity of a highly turbulent plasma," Phys. Rev. Lett., vol. 21, no. 10, pp. 674-\&, 1968.

[15] Y. Kawai and M. Guyot, "Observation of anomalous resistivity caused by ion-acoustic turbulence," Phys. Rev. Lett., vol. 39, no. 18, pp. 1141-1144, 1977.

[16] A. Hasegawa and T. Sato, "Existence of a negative potential solitary-wave structure and formation of a double-layer," Phys. Fluids, vol. 25, no. 4, pp. 632-635, 1982.

[17] K. Roberts and H. Berk, "Nonlinear evolution of a 2-stream instability," Phys. Rev. Lett., vol. 19, no. 6, pp. 297-\&, 1967.

[18] H. Pecseli and J. Trulsen, "A statistical-analysis of numerically simulated plasma turbulence," Phys. Fluids B: Plasma Phys., vol. 1, pp. 1616-1636, AUG 1989.

[19] G. Bonhomme, T. Pierre, G. Leclert, and J. Trulsen, "Ion phase-space vortices in ion-beam plasma systems and their relation with the ion-acoustic instability - numerical and experimental results," Plasma Phys. Contr. Fusion, vol. 33, pp. 507-520, MAY 1991.

[20] S. Borve, H. Pecseli, and J. Trulsen, "Ion phase-space vortices in 2.5-dimensional simulations," 
J. Plasma Phys., vol. 65, pp. 107-129, FEB 2001.

[21] B. Eliasson and P. Shukla, "The dynamics of electron and ion holes in a collisionless plasma," Nonlinear Proc. Geoph., vol. 12, no. 2, pp. 269-289, 2005.

[22] Y. Omura, H. Kojima, and H. Matsumoto, "Computer-simulation of electrostatic solitary waves - a nonlinear model of broad-band electrostatic noise," Geophys. Res. Lett., vol. 21, pp. 2923-2926, DEC 151994.

[23] P. Guio, S. Borve, L. Daldorff, J. Lynov, P. Michelsen, H. Pecseli, J. Rasmussen, K. Saeki, and J. Trulsen, "Phase space vortices in collisionless plasmas," Nonlinear Proc. Geoph., vol. 10, pp. 75-86, JAN-MAR 2003.

[24] G. Donoso, P. Martin, and J. Puerta, "Experimental-verification of the grid effects in a velocity analyzer with variable geometry," Rev. Sci. Instrum., vol. 57, pp. 1507-1511, AUG 1986.

[25] G. Donoso and P. Martin, "Space-charge effects in a velocity analyzer of variable geometry," Rev. Sci. Instrum., vol. 61, pp. 3381-3383, NOV 1990.

[26] H. Johnsen, H. Pecseli, and J. Trulsen, "Conditional eddies, or clumps, in ion-beam generated turbulence," Phys. Rev. Lett., vol. 55, no. 21, pp. 2297-2300, 1985.

[27] C. Franck, T. Klinger, and A. Piel, "Intermittency or noise - an experimental study on the ion-beam driven ion-acoustic instability," Phys. Lett. A, vol. 259, pp. 152-157, AUG 91999.

[28] K. Matyash, R. Schneider, F. Taccogna, A. Hatayarna, S. Longo, M. Capitelli, D. Tskhakaya, and F. X. Bronold, "Particle in cell simulation of low temperature laboratory plasmas," Contrib. Plasma Phys., vol. 47, no. 8-9, pp. 595-634, 2007.

[29] D. Gresillon and F. Doveil, "Normal modes in ion-beam - plasma system," Phys. Rev. Lett., vol. 34 , no. 2 , pp. $77-80,1975$.

[30] T. Fujita, T. Ohnuma, and S. Adachi, "Spatially growing waves in an ion beam-plasma system," Phys. Fluids, vol. 18, no. 9, pp. 1216-1218, 1975.

[31] Y. Kiwamoto, "Plasma turbulence generated by ion-ion 2-stream instability," J. Phys. Soc. Jpn., vol. 37, no. 2, pp. 466-474, 1974.

[32] Y. Nakamura, H. Bailung, and R. Ichiki, "Effects of a slow ion beam on ion-acoustic waves," Phys. Plasmas, vol. 11, pp. 3795-3800, AUG 2004.

[33] T. Ohnuma, T. Fujita, and Y. Hatta, "Oscillations in ion beam-plasma system," Phys. Lett. $A$, vol. A 36, no. 4, pp. 265-\&, 1971.

[34] Y. Saitou, Y. Nakamura, M. Tanaka, A. Komori, and Y. Kawai, "Experiments on suppression 
of ion-ion instability by RF pumps," Plasma Phys. Contr. Fusion, vol. 35, pp. 1755-1764, DEC 1993.

[35] R. Taylor and F. Coroniti, "Ion heating via turbulent ion-acoustic waves," Phys. Rev. Lett., vol. 29 , no. 1 , pp. $34-\&, 1972$.

[36] N. Sato, H. Sugai, and R. Hatakeyama, "Spatial evolution of velocity-modulated ion-beams in a plasma," Phys. Rev. Lett., vol. 34, no. 15, pp. 931-934, 1975.

[37] K. Sauer, "Fluid and kinetic ion acoustic dispersion relations.." Private Communications, 2011.

[38] A. Piel, H. Klostermann, A. Rohde, N. Jelic, and R. Schrittwieser, "Ion sheath oscillations in double plasma machines," Phys. Lett. A, vol. 216, pp. 296-302, JUN 241996.

[39] A. Sarma, H. Bailung, and J. Chutia, "Observation of beam-enhanced sheath instability in a double plasma device," Phys. Plasmas, vol. 3, pp. 3245-3250, SEP 1996.

[40] A. Rohde, H. Klostermann, and A. Piel, "Particle-in-cell simulation of grid sheath oscillations in a double-plasma device," IEEE T. Plasma. Sci., vol. 25, pp. 1144-1149, OCT 1997.

[41] C. Franck, O. Grulke, and T. Klinger, "Transition from unbounded to bounded plasma whistler wave dispersion," Phys. Plasmas, vol. 9, pp. 3254-3258, AUG 2002.

[42] Z. Harvey, S. C. Thakur, A. Hansen, R. Hardin, W. S. Przybysz, and E. E. Scime, "Comparison of gridded energy analyzer and laser induced fluorescence measurements of a two-component ion distribution," Rev. Sci. Instrum., vol. 79, OCT 2008. 17th Topical Conference on HighTemperature Plasma Diagnostics, Albuquerque, NM, 2008. 